\title{
FORMATION OF GALACTIC SYSTEMS IN LIGHT OF THE MAGNESIUM ABUNDANCE IN FIELD STARS: THE THICK DISK
}

\author{
V. A. Marsakov, T. V. Borkova \\ Institute of Physics, Rostov State University, \\ 194, Stachki street, Rostov-on-Don, Russia, 344090 \\ e-mail: marsakov@ip.rsu.ru, borkova@ip.rsu.ru \\ accepted 2005, Astronomy Letters, Vol. 31 No. 8, P.515-527
}

\begin{abstract}
The space velocities and Galactic orbital elements of stars calculated from the currently available high-accuracy observations in our summary catalog of spectroscopic magnesium abundances in dwarfs and subgiants in the solar neighborhood are used to identify thick-disk objects. We analyze the relations between chemical, spatial and kinematic parameters of $\mathrm{F}-\mathrm{G}$ stars in the identified subsystem. The relative magnesium abundances in thick-disk stars are shown to lie within the range $0.0<[\mathrm{Mg} / \mathrm{Fe}]<0.5$ and to decrease with increasing metallicity starting from $[\mathrm{Fe} / \mathrm{H}] \approx-1.0$. This is interpreted as evidence for a longer duration of the star formation process in the thick disk. We have found vertical gradients in metallicity $\left(\operatorname{grad}_{Z}[\mathrm{Fe} / \mathrm{H}]=-0.13 \pm 0.04 \mathrm{kpc}^{-1}\right)$ and relative magnesium abundance $\left(\operatorname{grad}_{Z}[M g / F e]=0.06 \pm 0.02 \mathrm{kpc}^{-1}\right)$, which can be present in the subsystem only in the case of its formation in a slowly collapsing protogalaxy. However the gradients in the thick disk disappear if the stars whose orbits lie in the Galactic plane but have high eccentricities and low azimuthal space velocities atypical of the thin-disk stars are excluded from the sample. The large spread in relative magnesium abundance $(-0.3<[\mathrm{Mg} / \mathrm{Fe}]<0.5)$ in the stars of the metal-poor "tail" of the thick disk which constitute $\approx 8 \%$ of the subsystem, can be explained in terms of their formation inside isolated interstellar clouds that interacted weakly with the matter of a single protogalactic cloud. We have found a statistically significant negative radial gradient in relative magnesium abundance in the thick $\operatorname{disk}\left(\operatorname{grad}_{R}[M g / F e]=-0.03 \pm 0.01 \mathrm{kpc}^{-1}\right)$ instead of the expected positive gradient. The smaller perigalactic orbital radii and the higher eccentricities for magnesium-richer stars, which among other stars, are currently located in a small volume of the Galactic space near the Sun are assumed to be responsible for the gradient inversion. A similar but statistically less significant inversion is also observed in the subsystem for the radial metallicity gradient.
\end{abstract}

Keywords: Galaxy (Milky Way), stellar chemical composition, thick disk, Galactic evolution.

\section{Introduction}

An insufficient accuracy of determining the ages of not only single stars, but also huge stellar ensembles, globular and open clusters, which are believed to consist of coeval stars, is a serious obstacle to reconstructing the formation history of the structure in the early 
Galaxy. Here, a "chemical" age indicator could be a help. According to present views, all of the chemical elements heavier than boron have been produced in nucleosynthesis reactions in stars of various masses. It thus follows that the number of atoms of heavy elements will inevitably increase as the Galaxy evolves, and the ratio of the number of atoms of heavy elements to the number of hydrogen atoms in the atmospheres of unevolved stars (i. e. their total metallicity) must serve as an indicator of their age. However numerous studies show that no age-metallicity relation can be traced for old Galactic populations convenient to use the relative abundances of various chemical elements instead of the total metallicity as a chemical age indicator of stars, since the formation of most elements can be attributed rather reliably to a particular nucleosynthesis process in stars of certain masses that evolve in a theoretically determined time. In particular high-mass type II supernovae (SNe II) are the main suppliers of $\alpha$-capture and r-process elements and a small amount of iron-group elements to the interstellar medium. In contrast, the bulk of the iron-group elements are synthesized in lower mass stars that are members of close binaries and that explode as type Ia supernovae (SNeIa). The $\alpha$-elements are produced in a shorter time than iron, because the evolution times of SNeII $(\approx 30 \mathrm{Myr})$ and $\mathrm{SNeIa}(\approx 1-2 \mathrm{Gyr})$ differ (see, e. g. Matteucci and Greggio 1986; Thielemann et al.1990; Tsujimoto et al.1995; Matteucci 2001)(although these authors believe that isolated, the very first SNe Ia could appear considerably earlier than this time only $\approx 0.5$ Gyr later). Since the contribution of SNe Ia to the synthesis of iron-group elements is significantly larger than their contribution to the synthesis of $\alpha$-elements, the $[\alpha / \mathrm{Fe}]$ ratio will decrease in the Galaxy as the interstellar medium is enriched with the remnants of these supernovae. Thus at least $\approx 1$ Gyr after the initial burst of star formation will pass by the time $[\alpha / \mathrm{Fe}]$ will begin to decrease.

It is well known that the boundary between the halo and the thick disk can be drawn through the point $[\mathrm{Fe} / \mathrm{H}] \approx-1.0$. Indeed, the distributions of subdwarfs, RR Lyrae variable stars, and globular clusters in heavy-element abundance exhibits a clear deficit of objects in the vicinity of precisely this point (see, e. g. Marsakov and Suchkov 1977; Zinn 1985; Borkova and Marsakov 2000, 2002). At the same time, there is evidence that the knee in the $[\alpha / \mathrm{Fe}]-[\mathrm{Fe} / \mathrm{H}]$ relation also occurs approximately at this location in our Galaxy. Never the less, there is no consensus about whether the relative abundance of $\alpha$-elements decreases with increasing total metallicity inside the thick disk. In particular having analyzed their rather small samples of stars, Gratton et al.(2000) and Fuhrmann (2000) argue that the abundance of $\alpha$-elements in thick-disk stars is approximately the same and decreases abruptly as one goes to thin-disk stars. At the same time, Prochaska et al.(2000) and Feldzing et al.(2003) derived the $[\alpha / \mathrm{Fe}]-[\mathrm{Fe} / \mathrm{H}]$ relation inside the thick disk from their small samples, with the knee occurring precisely at the point $[\mathrm{Fe} / \mathrm{H}] \approx$ -1.0. The difference between these two results is of fundamental importance in correctly reconstructing the formation history of the thick disk. Indeed if the thick disk were formed during the collapse of a protogalactic cloud then a trend in the relative abundance of $\alpha$ elements with increasing heavy-element abundance would inevitably arise in it (Prochaska et al. 2000). The thin disk could subsequently be formed in the Galaxy as a result of the ongoing collapse (see Burkert et al. 1992). The dificulty of this model lies in the fact that the total collapse time scale of the protogalactic cloud is much shorter than the evolution time scale of SNe Ia progenitors. This dificulty can be easily circumvented in a different class of models where the thick disk is formed from the very first thin-disk stars through the interaction of the Galaxy with a close satellite galaxy (see Kroupa 2002, Quinn et al. 1993). If further the stars of the newly forming thin disk are assumed to be formed from interstellar matter with additions of accreted intergalactic metal-poor gas, then the 
existence of metal-poor stars with thin-disk kinematics and the metallicity overlap between thick- and thin-disk stars in the range $-0.8<[\mathrm{Fe} / \mathrm{H}]<-0.3$ can be explained (Fuhrmann et al. 1995; Fuhrmann 1998). The dificulty of this model lies in explaining the existence of metal-rich globular clusters in the thick disk (Marsakov and Suchkov 1977). They cannot be heated as stars by a satellite galaxy and can appear only as a result of the star formation that accompanies this interaction (Gratton et al. 2000).

Magnesium is the best-studied $\alpha$-element since it has visible lines of various intensities and degrees of excitation in unevolved F-G stars. According to present views, almost all of the magnesium is synthesized in the envelopes of high-mass $\left(M>10_{\odot} M\right)$ presupernovae with hydrostatic carbon burning and is subsequently ejected into the interstellar medium by SNeII explosions. To perform a comprehensive statistical analysis of the chemical, physical, and spatial-kinematic properties of stellar populations in an effort to reconstruct the formation and evolution history of the Galactic subsystems, we compiled a summary catalog of spectroscopic magnesium abundances in stars with accurate parallaxes (Borkova and Marsakov 2005). In this catalog, we collected almost all of the magnesium abundances in dwarfs and subgiants in the solar neighborhood published in the past fifteen years and calculated the space velocity components and Galactic orbital elements for them. The size of our catalog is several fold larger than the size of any sample that has been used so far to analyze the chemical evolution of the Galaxy; this allows us to largely get rid of the observational selection that is often inherent in smaller samples. In this paper we consider the stellar population of the Galactic thick disk.

\section{INPUT DATA.}

In our compiled catalog the effective temperatures, surface gravities, and $[\mathrm{Fe} / \mathrm{H}]$ values for each star were obtained by averaging some 2000 determinations from 80 publications. The accuracies of the mean values of these quantities estimated from the scatters of the deviations of individual determinations about the calculated means for each star of the sample are $\varepsilon_{\text {eff }}= \pm 56^{\circ}$ and $\pm 82^{\circ}, \varepsilon \log g= \pm 0.12$ and \pm 0.24 , and $\varepsilon[\mathrm{Fe} / \mathrm{H}]= \pm 0.07 \mathrm{dex}$ and \pm 0.13 dex for stars with metallicities $[\mathrm{Fe} / \mathrm{H}]$ higher and lower than -1.0 , respectively. To avoid the uncertainties related to non-LTE effects for iron lines, we used everywhere the iron abundances determined solely from Fe II lines. Since the non-LTE corrections for magnesium in F-G dwarfs and subgiants are small and do not exceed 0.1 dex (Shimanskaya et al. 2000), they were not considered separately in our catalog. The relative magnesium abundances were derived from 1412 determinations in 31 publications for 867 dwarfs and subgiants by using a three-pass iterative averaging procedure with the assignment of a weight to each primary source and to each individual determination. The systematic shifts of all scales relative to the reduced mean scale were taken into account. Assigning the lowest weight to the least reliable determinations, this procedure yields final values close to those given by most sources without excluding any determination. The internal accuracies of the $[\mathrm{Mg} / \mathrm{Fe}]$ values for metal-rich and metal-poor stars are $\varepsilon[\mathrm{Mg} / \mathrm{Fe}]= \pm 0.05$ dex and \pm 0.07 dex respectively.

The distances to the stars and their proper motions were calculated using data from currently available high-accuracy catalogs, both orbital (Hipparcos - Tycho) and ground-based (PPM-N, PPM-S, PPM-add) ones. We used the trigonometric parallaxes with errors of less than $25 \%$ and, in the absence of the latter the published photometric distances determined from uvbyb photometry. The radial velocities were taken mostly from the catalogs by Nidever et al.(2002), Barbier-Brossat and Figon (2000), and Nordstrem et al.(2004). In 
the absence of necessary data in these catalogs, they were taken from other sources (see the references to Table 2 in our previous paper (Borkova and Marsakov 2005)).

The components of the total space velocity $(U, V, W)$ relative to the Sun were calculated for 844 stars with measured distances, proper motions, and radial velocities, where $U$ is directed toward the Galactic anticenter $\mathrm{V}$ is directed in the sense of Galactic rotation, and $\mathrm{W}$ is directed toward the North Galactic Pole. At mean errors in the distances of $15 \%$ and a mean heliocentric distance of the stars from our sample of $\approx 60 \mathrm{pc}$, the mean error in the space velocity components was $\approx \pm 2 \mathrm{~km} \mathrm{~s}^{-1}$.

Based on the multicomponent model of the Galaxy containing a disk, a bulge, and an extended massive halo (Allen and Santillan 1991), we computed the Galactic orbital elements by simulating 30 revolutions of a star around the Galactic center. The Galactocentric distance of the Sun was assumed to be $8.5 \mathrm{kpc}$, the rotational velocity of the Galaxy at the solar Galactocentric distance was $220 \mathrm{~km} \mathrm{~s}^{-1}$, and the velocity of the Sun with respect to the local standard of rest is $\left(U_{\odot}, V_{\odot}, W_{\odot}\right)=(-11,14,7.5) \mathrm{km} \mathrm{s}^{-1}$ (Ratnatunga et al.1989).

For our studies, we retained only 847 dwarfs and subgiants in the solar neighborhood with $T_{\text {eff }}>5000 \mathrm{~K}$; in cooler stars, the elemental abundances could be distorted. For 545 stars of our sample, we used the ages determined from theoretical isochrones in the paper by Nordstrem et al.(2004). The absolute magnitudes of the stars were found in this paper as in our paper mainly from the trigonometric parallaxes (from the photometric distances for some of the stars with large errors in the parallaxes), while the metallicities were derived from uvby photometry. A check shows that their photometric metallicities and temperatures are in good agreement with our spectroscopic data (the mean deviation is $\delta\left([\mathrm{Fe} / \mathrm{H}]_{p f}-[\mathrm{Fe} / \mathrm{H}]_{s p}\right)=0.07$ dex, for the iron abundance at the dispersion of the deviations $\sigma_{\delta[\mathrm{Fe} / \mathrm{H}]}=0.09 \mathrm{dex}$; and $\delta T=45^{\circ}$ for the temperature at $\sigma_{\delta T}=70^{\circ}$ i. e., of the order of the above errors in the corresponding quantities in our catalog), which allows us to use the ages from this paper. The absolute values of the age errors given by the authors are rather large: the error is less than 1 Gyr only for $\approx 30 \%$ of the stars, ranges from 1 to 2 Gyr for another $\approx 25 \%$ of the stars, and is even larger up to $\approx 7$ Gyr for the remaining stars.

All stars of our sample fill the entire sky rather uniformly. Nevertheless, it exhibits observational selection: the apparent magnitude depends on metallicity (see Fig. 7 in our previous paper (Borkova and Marsakov (2005)). On the one hand, it stemmed from the fact that mostly metal-richer stars lie near the Galactic plane (where the Sun is located). On the other hand, observers knowingly use more sensitive instrumentation to increase the number of studied metal-poorer stars. As a result, almost all of the stars with $[\mathrm{Fe} / \mathrm{H}] \geq-1.0$ were brighter than $V \approx 9^{m}$ while the bulk of the metal-poorer stars were fainter. However our results are not affected by this selection.

\section{IDENTIFICATION OF STARS OF THE SUBSYSTEMS}

There is no single, necessary and sufficient criterion that would allow each star to be individually identified with absolute confidence by its belonging to a particular Galactic subsystem. Only the mean values and dispersions of such key parameters of the stellar subsystems as the ages, velocities, spatial locations, Galactic orbital elements, metallicities, and relative abundances of certain chemical elements can be determined more or less accurately from reliably identified members of the subsystem. Any of the above parameters can act as a criterion for identifying the stars of various subsystems. For globular star 
clusters, the morphological structure of their horizontal branches and the mean periods of their RR Lyrae stars (cf. Oosterhofs types) can also be attributed to these determining parameters. Choosing any of these parameters, we get an opportunity to study the distributions of the subsystems objects in other parameters. Such studies have revealed that no sharp boundaries exist between the subsystems and that stars with identical characteristics can belong to different subsystems. It would be appropriate to use complex criteria that include several parameters to identify the subsystems objects more reliably.

The metallicity of stars is commonly used to separate the stars of the spherical Galactic subsystems from the diskones, since it remains, though rough, an age indicator. However in this paper we chose exclusively kinematic parameters as the initial criteria, since they shape the spatial distribution of objects that manifests itself in the morphological identification of several subsystems in the Galaxy. In this approach the possibility of observational selection that distorts the abundance distributions of various chemical elements in the stars of the subsystems, particularly in the near-boundary regions, is ruled out. We separated the thick-disk stars from the halo using the following condition: $V_{\text {res }}=\sqrt{\left(U^{2}+V^{2}+W^{2}\right)}<$ $175 \mathrm{~km} \mathrm{~s}^{-1}$, where $U, V, W$ and $V_{\text {res }}$ are the space velocity components and the total residual stellar velocity relative to the local standard of rest, respectively. We optimized the specific value of this criterion by minimizing the numbers of metal-rich $([\mathrm{Fe} / \mathrm{H}]>-1.0)$ sample stars in the identified halo and metal-poor stars in the thick disk. The derived critical value of the residual velocity for the selection of thick-disk stars from our sample is almost equal to that in the paper by Fuhrmann (2000): $V_{\text {res }}=180 \mathrm{~km} \mathrm{~s}^{-1}$. Chiappini et al. (1997) suggested a fairly informative kinematic index based on the same three space velocity components or more precisely on the Galactic orbital elements of stars calculated from them. This is $\sqrt{Z_{\max }^{2}+4 \cdot e^{2}}$, where $Z_{\max }$ is the maximum distance of the orbital points from the Galactic plane, and is the orbital eccentricity of the star. Figure 1a shows the relation between these two kinematic indices. Even a distinct gap between the halo and the thick disk, which is indicated by the dotted line, is observed in the diagram (however it could also be the result of selection in the original lists of stars used to compile the summary catalog).The identified subsystems are also naturally separated in the $[\mathrm{Fe} / \mathrm{H}]-$ $\sqrt{Z_{\max }^{2}+4 \cdot e^{2}}$ plane, i. e. composed of the two independent criteria that we did not use,and form two clusters that can be arbitrarily separated (see the dotted line in Fig. 1b). The stars lying in the immediate vicinity of this demarcation line occupy controversial positions here. However according to our kinematic criterion almost all of them fall into the thick disk as can be seen from the diagram. As a checkshows, they fill rather uniformly the same regions in all kinematic diagrams of Fig. 1 as the remaining thick-disk stars. However excluding these stars from the thick disk would introduce artificial chemical selection into both separated systems, and a certain number of stars with an intermediate metallicity and with kinematics typical of the thick-disk stars would be attributed to the halo. Very metal-poor but rapidly revolving (around the Galactic center) stars are commonly called the metal-poor tail of the thick disk (Prochaska et al. 2000). We singled out 13 stars with $[\mathrm{Fe} / \mathrm{H}]<-1.25$ from the kinematically selected thick-disk objects in order to subsequently trace the correspondence of their properties to those of the subsystems remaining stars. The metal-poor tail stars are denoted in Fig. 1 by the open triangles. Figure 1c shows the $\Theta-\sqrt{Z_{\max }^{2}+4 \cdot e^{2}}$ diagram, where $\Theta$ is the circular velocity of the star around the Galactic center. We see from this figure that the halo and thick-disk stars (except several stars) in this diagram are separated by a clear gap, while the metal-poor tail stars fill uniformly the region occupied by all of the remaining thick-disk stars. The location of the gap is highlighted in the diagram by the inclined dotted line. The distribution of 
stars of the identified subsystems in Toomre's diagram is shown in Fig. 1d. We emphasize that any criterion is purely statistical and the membership of each specific star remains doubtful. For example, according to the residual velocity criterion, the star HD 97916 falls into the thick disk but its high value of $Z_{\max }>4 \mathrm{kpc}$, which is atypical of the stars of this subsystem (see Fig. 6 below), suggests that it can also belong to the halo with equal success (see Fig. 1). Based on the $3 \sigma$ criterion applied to the parameter $Z_{\text {max }}$, we excluded this star from the subsequent analysis among the thick-disk stars.

The thin-disk stars are known to have low residual velocities with respect to the local standard of rest and nearly circular orbits all points of which do not rise high above the Galactic plane. Therefore, for reliability we identified this subsystem using simultaneously two kinematic criteria: $V_{\text {res }}<85 \mathrm{~km} \mathrm{~s}^{-1}$ (see also Furmann 1998) and $\sqrt{Z_{\max }^{2}+4 \cdot e^{2}}<$ 1.05. Higher-velocity stars were included in the thick disk. In this case, the values of the criteria were chosen to minimize the number of stars with a high relative abundance of magnesium in the thin disk and with its low abundance in the thick disk. It turned out that we could not get rid of the overlap between the abundance ranges of various chemical elements in the stars of the subsystems being separated by any combination of

any kinematic parameters. However the $V_{\text {res }}-\sqrt{Z_{\max }^{2}+4 \cdot e^{2}}$ diagram (Fig. 1a) reveals even sparseness of stars between the subsystems. Figures 1a and 1d show that we cannot reliably determine which subsystem a star belongs to from any single velocity component. Indeed, according to the adopted criteria, a star in the thick disk can have any arbitrarily low velocity component, but the other velocity components (or only one component) will necessarily be appreciably higher than those for the disk stars.

If we follow the kinematic criterion then the metallicity is represented by not quite a proper criterion. Indeed, a significant overlap of the metallicity ranges between the thick and thin disks is seen from Fig. 1b. Consequently the criterion based on the position of the dip found in the metallicity function of field stars near $[\mathrm{Fe} / \mathrm{H}]=-0.5$ (see, e. g. Marsakov and Suchkov 1977), which is commonly used to separate the stars of the subsystems, can be used only when there are no data on the total space velocities of the stars. The situation with the age is also difficult. Based on the accurately determined ages of only seven subgiants, Bernkopf et al. (2001) concluded that the thin disk began to form $\approx$ 9 Gyr ago, while the thick-disk stars are definitely older than 12 Gyr. Nevertheless, the ages, unfortunately cannot yet be used as a reliable criterion for separating the two disk subsystems, because the individual ages of most stars are determined with a low accuracy. Therefore in this paper we use the ages only to reveal statistical trends.

\section{RELATION BETWEEN THE IRON AND MAGNESIUM ABUNDANCES}

Let us consider the properties of the stars of the thick disk that we identified. Elucidating the question of whether there is a trend in the relative magnesium abundance with metallicity inside the thick diskis of greatest importance here. Figure 2a shows the $[\mathrm{Mg} / \mathrm{Fe}]-[\mathrm{Fe} / \mathrm{H}]$ diagram for all stars of our catalog where the identified thick-disk stars are highlighted by the triangles. To checkthe results using more reliable data, exactly the same diagram, but only for the stars in which the magnesium abundances were averaged over several sources and with the total weight of the mean value larger than unity is shown in Fig. 2b. Figure 2c shows the stars of only the thick disk and plots the median curve that roughly fits the pattern of $[\mathrm{Mg} / \mathrm{Fe}]$ variation with increasing metallicity. Unfortunately this dependence cannot be constructed mathematically rigorously; therefore, we 
drew it by eye halfway between the upper and lower envelopes of the diagram. We see from the figure that an appreciable number of stars with thick-disk kinematics have a chemical composition typical of the thin-disk stars, with the magnesium trend with increasing metallicity for these stars being very similar to that in the thin disk. Most stars (9 of the 13) of the metal-poor thick-disk tail (open triangles) show approximately the same magnesium abundance, $\langle[\mathrm{Mg} / \mathrm{Fe}]\rangle=0.40 \pm 0.01$ but some of them (four stars) at metallicity $[\mathrm{Fe} / \mathrm{H}]<-1.7$ exhibit a very low abundance ratio, $[\mathrm{Mg} / \mathrm{Fe}]=(-0.3 \div 0.3)$ dex which is atypical of the stars formed from matter with the same chemical evolution. Starting from $[\mathrm{Fe} / \mathrm{H}] \approx-1.0$ (the vertical dotted line in the figure), the tendency for the relative magnesium abundance to decrease with increasing metallicity begins to show up increasingly clearly in the thick disk. Even if we restrict our analysis to the magnesium abundance range $[\mathrm{Mg} / \mathrm{Fe}]>0.25$ the correlation coefficient turns out to be larger than zero beyond the error limits. However even when the boundary is lowered to $[M g / F e]=0.20$, i. e. to the upper limit for the overwhelming majority of thin-disk stars, the correlation coefficient becomes equal to $r=0.4 \pm 0.1$ (see the inclined straight lines in Figs. 2a and 2b). If, however, we include metal-poorer thick-disk star up to $[\mathrm{Fe} / \mathrm{H}]=-1.25$ then the slopes of the regression lines in both diagrams will decrease slightly. However the figure shows a considerable number of thick-disk stars with even lower magnesium abundances, and the correlation coefficient increases to $r=0.65 \pm 0.05$ for all of the thick-disk stars with metallicities $[\mathrm{Fe} / \mathrm{H}]>-1.0$. This increase in correlation is attributable mainly to a sudden decrease in the mean magnesium abundance starting from $[\mathrm{Fe} / \mathrm{H}] \approx-0.6$ which showed up as a sharp bend of the median sequence in Fig. 2c. The sequence again becomes flatter starting from $[\mathrm{Fe} / \mathrm{H}] \approx-0.35$. Thus the data from our catalog suggest that the $[\mathrm{Mg} / \mathrm{Fe}]$ ratio decreases with increasing $[\mathrm{Fe} / \mathrm{H}]$ in the thick disk and that the explosions of the first SNe Ia began when the mean metallicity of the interstellar medium in the Galaxy reached $[\mathrm{Fe} / \mathrm{H}] \approx-1.0$. Howeverthe explosions of SNe Ia began later than the mass star formation in the thick disk which occurred (as can be seen from Fig. 2) even at $[\mathrm{Fe} / \mathrm{H}] \approx-1.25$. These results confirm the conclusion about a fairly long duration of the star formation in the subsystem that was drawn by Prochaska et al. (2000) from the trends in three other $\alpha$-elements with metallicity detected by the authors. Prochaska et al. (2000) argue that such a long time scale is possible only in the thick-disk formation scenario during dissipational collapse. The formation of metal-poor tail stars in this scenario can be explained by the fact that a number of stars with kinematics characteristic of the thick-disk stars were formed from isolated clouds of interstellar matter less enriched with heavy elements. The existence of stars with a low magnesium abundance $([\mathrm{Mg} / \mathrm{Fe}]<0.2 \mathrm{dex})$ in the metal-poor tail at low heavy-element abundances most likely implies that these clouds were in weak contact with the bulk of the protogalactic cloud in which the matter was mixed fairly well.

A further argument for the latter suggestion is the result by Navarro et al. (2004). They provide evidence that the stars of the long known Arcturus moving group (see Eggen 1998) may well originate from a massive satellite disrupted by the Galactic tidal forces at an early formation phase of the Galaxy. We selected stars with close angular momenta from the original catalog according to the criteria in the paper by Navarro et al.(2004): $(85<\Theta<$ 130), $|W|<50^{-1}$ and $|\Pi|<150^{-1}$, where $\Pi, \Theta$, and $W$ are the space velocity components of the stars with respect to the local standard of rest in the cylindrical coordinate system. It turned out that 15 of the 17 stars selected in this way belong to our thick disk and 3 of them belong to its metal-poor tail (two stars fell into the protodisk halo). Figure 2 shows the $[\mathrm{Mg} / \mathrm{Fe}]-[\mathrm{Fe} / \mathrm{H}]$ diagram for the thick-disk stars (triangles); the circles denote the stars of the Arcturus group. The considerably smaller spread in $[\mathrm{Mg} / \mathrm{Fe}]$ ratios than that for 
the thick-disk stars at given metallicity for Arcturus group candidates argues that these stars may well have a common origin. Thus we see that at least some of the stars with thick-disk kinematics can be extragalactic in origin, i. e. be formed from matter with a different history of chemical evolution.

All of the currently existing thick-disk formation scenarios can be divided into two classes:(1) the collapse of a single protogalactic cloud; and (2) the result of the interaction between the early Galaxy and nearby satellite galaxies. The behavior of the subsystems stars in different scenarios must differ. Thus, for example, according to the theoretically modeled first scenario a vertical gradient in chemical composition must be formed in the thick disk through the collapse of the protogalactic cloud (Burkert et al. 1992). However free collapse takes a very short time, $\approx 400$ Myr while the relative abundances of chemical elements in the interstellar medium can change appreciably only on much longer time scales. The above evidence for the existence of SNe Ia traces in the chemical composition of the subsystems stars suggests that the overwhelming majority of stars in it were formed after the mass explosions of supernovae of this type began, i. e. no earlier than $12 \mathrm{Gyr}$ (see, e. g. Matteucci 2001) after the initial star formation in the protogalactic cloud. By this time according to the hypothesis of rapid collapse, all of the interstellar matter in the protogalaxy had already concentrated near the plane. This contradiction is naturally circumvented in the theoretical model where as the result of a close encounter with a fairly massive satellite galaxy the already formed stars of the primordial thin disk acquire a significant acceleration mainly perpendicular to the Galactic plane, producing the thick disk (Kroupa 2002). In addition, at all evolutionary phases, the tidal forces of the early Galaxy could disrupt dwarf satellite galaxies, capturing their stars, and as predicted by the corresponding theoretical model (Quinn et al. 1993), it is then unlikely that a vertical gradient in chemical composition will arise in its thick disk. The latter assumption is supported by the possible extragalactic origin of the Arcturus moving group, almost all the stars of which belong to the thick disk.

To concretize the model, let us analyze the relation between the chemical composition and other parameters of the subsystems stars.

\section{RELATION OF THE IRON AND MAGNESIUM ABUN- DANCES TO ORBITAL ELEMENTS AND AGE}

Having reconstructed the Galactic orbits of the stars, we can trace the variations in metallicity and relative magnesium abundance in the subsystem with maximum distance of the stars from the Galactic center and plane. Figure 3a shows the $Z_{\max }-[\mathrm{Fe} / \mathrm{H}]$ diagram for all thick-disk stars. The thirteen metal-poor tail stars with $[\mathrm{Fe} / \mathrm{H}]<-1.25$ are highlighted by the open triangles and are not used to determine the gradients. The regression line constructed for the remaining stars exhibits a fairly large negative vertical metallicity gradient: $\operatorname{grad}_{Z}[\mathrm{Fe} / \mathrm{H}]=(-0.13 \pm 0.04) \mathrm{kpc}^{-1}$ at $r=-0.27 \pm 0.08$. The result is stable, since even eliminating the four most distant stars lying farther than $2 \mathrm{kpc}$ do not change the slope of the regression line at all. The correlation is highly significant, since the probability of random occurrence of the correlation is $P_{N} \ll 1 \%$. The vertical gradient in the thick disk derived from the nearest stars is equal, within the error limits, to the value of $-0.2 \pm 0.1 \mathrm{kpc}^{-1}$ obtained from $\mathrm{RR}$ Lyrae variable field stars (see Borkova and Marsakov 2002). The thick-disk stars also exhibit a vertical gradient in relative magnesium abundance (see Fig. 3b): $\operatorname{grad}_{Z}[\mathrm{Mg} / \mathrm{Fe}]=(0.06 \pm 0.02) \mathrm{kpc}^{-1}$ at $r=0.33 \pm 0.08$ and at a probability of random occurrence of the correlation $P_{N} \ll 1 \%$; i. e., the correlation can 
be recognized to be highly significant. Here,removing the four most distant stars changes neither the correlation coefficient nor the gradient. Note that the correlations in Figs. 3a and $3 \mathrm{~b}$ stem exclusively from the existence of two groups of points that are separated by a distinct deficit of stars near $Z_{\max } \approx 0.5 \mathrm{kpc}$; it is not quite clear why it arose (see also the $Z_{\text {max }}$ distribution of stars in Fig. 6a). There are no correlations inside each group but they differ in both mean magnesium abundance and metallicity. Therefore, if we used a more stringent criterion and did not include low-orbit stars in the subsystem (in Fig. 1 this roughly corresponds to the satisfaction of the condition $\sqrt{Z_{\max }^{2}+4 \cdot e^{2}}>0.8$ for the thickdisk), then the two vertical gradients in it would disappear. However the high orbital eccentricities $(\langle e\rangle=0.33 \pm 0.01)$, residual velocities $\left(\left\langle V_{\text {res }}\right\rangle=97 \pm 2 \mathrm{~km} \mathrm{~s}^{-1}\right)$, and ages $(\langle t\rangle=9.7 \pm 0.9 \mathrm{Gyr})$ and the low azimuthal space velocity $\left(\langle\Theta\rangle=190 \pm 6 \mathrm{~km} \mathrm{~s}^{-1}\right)$ even at low magnesium abundance $([\mathrm{Mg} / \mathrm{Fe}]=0.21 \pm 0.02)$ in the excluded stars do not allow them to be attributed to the thin disk. Therefore some of the authors (e. g., Fuhrmann 1998; Bensby et al. 2003) did not include them in any of the disk subsystems, calling them transitional stars. Thus the question as to whether a vertical gradient in chemical composition exists in the thick disk will be solved only after it will be established which subsystem this group of stars belongs to.

The radial metallicity gradient in the thick disk (Fig. 3c) was found to be positive rather than negative, but almost indistinguishable from zero outside the error limits $(r=$ $0.18 \pm 0.09)$. Since the probability of random occurrence of this correlation is $P_{N}=5 \%$ it cannot be recognized to be statistically significant. The RR Lyrae variable stars in the thick disk reveal no radial metallicity gradient at all (Borkova and Marsakov 2002). In contrast, the radial gradient in relative magnesium abundance is not only nonzero outside the $3 \sigma$ limits, but also is negative, instead of the expected increase in the relative magnesium abundance with increasing apogalactic orbital radius of the stars: $\operatorname{grad}_{R_{a}}[\mathrm{Mg} / \mathrm{Fe}]=(-$ $0.026 \pm 0.007) \mathrm{kpc}^{-1}$ at $r=-0.34 \pm 0.08$ and at a probability of random occurrence of the same correlation $P_{N} \ll 1 \%$ (see Fig. $3 \mathrm{~d}$ ). Note that the corresponding radial gradients and correlation coefficients in Figs. 3e and $3 \mathrm{f}$ were found to be almost the same when using the mean orbital radii of the stars. The latter are believed to be less subject to variations during the stellar lifetime and therefore, are commonly used to find the initial radial metallicity gradients. Remarkably eliminating the stars with $\sqrt{Z_{\max }^{2}+4 \cdot e^{2}}<0.8$ from the subsystem has virtually no effect on the results, since the stars with any orbital radii are eliminated more or less equiprobably. We clearly see from the diagrams that the density of stars with a high magnesium abundance increases continuously with decreasing galactocentric distance and the overwhelming majority of them are found near the solar orbital radius, while the density of magnesium-poor stars in the diagrams is almost independent of both $R a$ and $\langle R\rangle$. Therefore,even if we reject the seven most distant stars in the diagrams (with $R a>13 \mathrm{kpc}$ in Fig. 3d and with $\langle R\rangle>10 \mathrm{kpc}$ in Fig. 3f), both the slopes of the regression lines and the correlation coefficients will only increase. The relative numbers of stars with different metallicities in the $[\mathrm{Fe} / \mathrm{H}]-\mathrm{Ra}$ (Fig. 3c) and $[\mathrm{Fe} / \mathrm{H}]-\langle R\rangle$ (Fig. 3e), where positive correlations are observed instead of the expected negative correlation behave in a similar way. This gradient inversion is most likely caused by the selection effect related to the predominantly smaller perigalactic orbital radii and larger eccentricities for the metal-poorer, and simultaneously magnesium-richer stars in the solar neighborhood (see the correlations in the diagrams of Fig. 4). Indeed the probability of finding a star near its apogalactic orbital radius is highest. Therefore among the thick-disk stars with fairly high orbital eccentricities $\left(\langle e\rangle=0.34\right.$ at $\left.\sigma_{e}=0.13\right)$, we will see a larger fraction of slow stars near the apogalactic radius equal to the solar orbital radius than stars with large $R a$ and 
$\langle R\rangle$ passing rapidly by the Sun. At the same time, the relative velocities for the stars with the perigalactic radii equal to the solar orbital radius are low; therefore, among the stars with large $R a$ and $\langle R\rangle$ in the solar neighborhood, the fraction of such stars is larger. Since both the magnesium abundance $\left(r=-0.44 \pm 0.07\right.$ at $\left.P_{N} \ll 1 \%\right)$, and to a small degree, the metallicity $\left(r=0.18 \pm 0.09\right.$ at $\left.P_{N} \ll 5 \%\right)$ in the thick disk correlate with $R p$ (see Figs. 4a and 4c, respectively), magnesium-poorer, and simultaneously metal-poorer stars will prevail at large $R a$. The correlation between magnesium abundance and eccentricity $\left(r=0.34 \pm 0.08\right.$ at $\left.P_{N} \ll 1 \%\right)$ in Fig. 4b also contributes to the enhancement of this selection. In Fig. 4d metallicity does not correlate with eccentricity; nevertheless, its sign is negative: $r=-0.11 \pm 0.09$ at $P_{N} \ll 28 \%$. We emphasize that both correlations with the magnesium abundance should be recognized to be highly significant and eliminating the low-orbit stars from the subsystem does not change the result. Thus, the usually declared absence of a radial metallicity gradient and the existence of the negative (inverse) radial magnesium abundance gradient in the thick disk found above can be largely attributed to the selection related to the observations of stars in a limited region of Galactic space.

The distributions of the stars that are currently in the local volume of the Galaxy in orbital eccentricity and maximum distance from the Galactic plane are less dependent on the observers location, i. e. the Sun than their distributions in maximum and minimum Galactocentric orbital radii. Therefore, these orbital elements may be expected to also correlate with stellar age. We see from Fig. 5 that the two parameters indeed exhibit a correlation with age. However the $e(t)$ correlation is actually significant (the correlation coefficient is $r=0.35 \pm 0.10$ at $P_{N} \ll 2 \%$ ), while the $\left(\mathrm{t}-Z_{\max }\right)$ diagram exhibits no correlation but it clearly shows the absence of large distances from the Galactic plane among the young stars (see the empty upper left corner in Fig. 5b separated by the dotted line). The magnesium abundance also correlates with age $\left(r=0.27 \pm 0.11\right.$ at $P_{N} \ll 2 \%$ i. e., the correlation should still be recognized to be significant), while the metallicity does not correlate with age at all (see Figs. 5c and 5d,respectively). Eliminating the low-orbit stars changes nothing. Let us $[\mathrm{Mg} / \mathrm{Fe}]-[\mathrm{Fe} / \mathrm{H}]$ consider the $\mathrm{t}-[\mathrm{Mg} / \mathrm{Fe}]$ diagram in more detail. According to Fuhrmann (2000), there can be no stars younger than $\approx 10$ Gyr in the thick disk but they are present in our diagram. The larger triangles in the diagram denote the stars with age errors of less 2 Gyr. We see that the number of stars decreased by a factor of 2 but the overall structure of the diagram did not change and the correlation was retained. The stars in the diagram are naturally separated into two groups by the dotted line drawn almost to the regression line. The groups of different ages turned out to differ in both mean space velocity and galactic orbital elements. In particular their mean residual velocities $\left\langle V_{\text {res }}\right\rangle=(95 \pm 3) \mathrm{km} \mathrm{s}^{-1}$ and $(116 \pm 3) \mathrm{km} \mathrm{s}^{-1}$, their dispersions $\left\langle\sigma_{V_{\text {res }}}\right\rangle=(13 \pm 2) \mathrm{km} \mathrm{s}^{-1}$ and $25 \pm 2 \mathrm{~km} \mathrm{~s}^{-1}$, azimuthal velocities $\langle\Theta\rangle=(200 \pm 7) \mathrm{km} \mathrm{s}^{-1}$ and $156 \pm 4 \mathrm{~km} \mathrm{~s}^{-1}$, orbital eccentricities $\langle e\rangle=(0.31 \pm 0.01)$ and $0.36 \pm 0.01$, and the maximum distances of orbital points from the Galactic plane $\left\langle Z_{\max }\right\rangle=(0.55 \pm 0.10) \mathrm{kpc}$ and $0.87 \pm 0.07 \mathrm{kpc}$, respectively for the young and old groups. A subgroup of six stars with a high magnesium abundance (the metal-poor tail stars were not included) stands somewhat apart from the stars with young ages. The mean values of its kinematic parameters listed above are almost equal to those for the main subgroup of young stars. The subgroups differ only in magnesium abundance and metallicity: for the subgroup of six stars, $[\mathrm{Fe} / \mathrm{H}]=-0.74 \pm 0.10 \mathrm{dex}$ is almost equal to the metallicity of the old group $-0.63 \pm 0.03$ dex while for the main young subgroup, it is $-0.35 \pm 0.05$ dex. The fact that 8 of the 24 stars in the young magnesium-poor subgroup have a circular velocity $\Theta>220 \mathrm{~km} \mathrm{~s}^{-1}$ and $R a>12 \mathrm{kpc}$ was unperpendicularly expected. There are virtually no such rapidly revolving stars in the old group. Judging 
by their characteristics, these stars could well be attributed to the thin disk if it were not for the larger $Z_{\max }>1 \mathrm{kpc}$ for half of them, and $e>0.3$ which is a typical of the thin-and disks tars, for the remaining stars. Thus, it seems that the thick disk consists of two discrete populations that differ in ages, elemental abundances, angular momenta, space velocity dispersions, and orbital elements. However the belonging of the young group to the thick disk requires additional proofs.

The scale height is one of the most important parameters of the subsystem. It characterizes the subsystems thickness and is equal to the height above the Galactic plane at which the density of its objects decreases by a factor of $e$. This parameter can also be determined from nearby stars by constructing their $Z_{\max }$ distribution and fitting it by an exponential law. The following should be taken into account: first, the kinematic selection, since the probability of detecting a star at a given time near the Sun decreases with its increasing relative velocity; and, second, we must not just include each star in the histogram with its $Z_{\max }$ but as it were, "spread" it over the orbit from $-Z_{\max }$ to $+Z_{\max }$ proportional to the probability density of finding it at diferent $Z$. This is because all stars of the subsystem cannot be simultaneously at the maximum distances from the Galactic plane. To allow for the kinematic selection, we assigned a weight to each star proportional to its residual velocity with respect to the local standard of rest, $V_{\text {res }}$. The initial $Z_{\max }$ histogram and the reconstructed actual $Z$ distribution of thick-disk stars are shown in Fig. 6. The solid curve in Fig. 6b represents a function of the form $n(Z)=C \cdot \exp \left(-Z / Z_{0}\right)$ where $Z_{0}$ is the scale height (for more details on the procedure for reconstructing the height distribution of stars, see Marsakov and Shevelev (1995)). The scale height for the thick disk was found from the stars of our sample to be $Z_{0}=0.6 \pm 0.1 \mathrm{kpc}$, where the uncertainty was estimated from the maximum and minimum values of $Z_{0}$ obtained by eliminating the extreme columns of the histogram. The derived scale height is slightly smaller than its value that we determined also from the RR Lyare variable field stars: $Z_{0}=0.74 \pm 0.05 \mathrm{kpc}$ (see Borkova and Marsakov 2002), while the latter matches the estimates of other authors (in particular Robin et al. (1996) gave a value averaged over several source of $Z_{0}=0.76 \pm 0.05 \mathrm{kpc}$ ). The size of the sample used must be increased significantly to reliably estimate the scale height for the subsystem. Note also that the scale height slightly increases if the low-orbit stars are eliminated from the subsystem.

The mean velocity components and their dispersions calculated from all 133 thick-disk stars of our sample are: $\langle U\rangle=(4 \pm 7) \mathrm{km} \mathrm{s}^{-1} \sigma_{U}=(80 \pm 5) \mathrm{km} \mathrm{s}^{-1} ;\langle V\rangle=(-49 \pm 4) \mathrm{km} \mathrm{s}^{-1}$ $\sigma_{V}=(46 \pm 3) \mathrm{km} \mathrm{s}^{-1} ;\langle W\rangle=(2 \pm 4) \mathrm{km} \mathrm{s}^{-1} \sigma_{W}=(45 \pm 3) \mathrm{km} \mathrm{s}^{-1}$. They are in satisfactory agreement with the determinations of other authors even in the case of a radical difference between the initial prerequisites in forming the samples of thick-disk stars. For example, Soubiran et al. (2003) obtained $\left(\sigma_{U}, \sigma_{V}, \sigma_{W}\right)=(63 \pm 6,39 \pm 4,39 \pm 4) \mathrm{km} \mathrm{s}^{-1}$ for a sample of $\approx 400$ thick-disk red giants lying toward the North Galactic Pole. On the other hand, Gilmore and Wyse (1987) give the following velocity dispersions for the thick-disk field stars: $(70,50,45) \mathrm{km} \mathrm{s}^{-1}$.

\section{THE FORMATION TIME SCALE OF THE THICK DISK}

Thus the trends that we found here strongly suggest that the formation time scale of the thick disk was rather long and in no way fits into the scenario of a rapidly collapsing protogalaxy (Eggen et al. 1962). The formation of the first stars in it began long before the onset of mass type-Ia supernovae explosions when the metallicity had not yet reached a turning value of $[\mathrm{Fe} / \mathrm{H}] \approx-1.0$. Since the evolution time scale of close binary stars that 
end their lives with SNe Ia explosions is $\approx(1-2)$ Gyr the formation of the subsystem began before the Galaxy reached this age. By this time, the young Galaxy already had an almost formed spherical subsystem. By studying the variations in the abundances of $\mathrm{r}-$ and s-elements in thick-disk stars with magnesium abundance $[\mathrm{Mg} / \mathrm{Fe}]>0.25$ Mashonkina et al. (2003) showed that the overwhelming majority of thick-disk stars were formed within 1.1 to 1.6 Gyr after the protogalactic cloud began to collapse. However the last stars in the thick disk turned out to have been born after the formation of a younger subsystem, the thin disk, began. This is evidenced by our largely selection-free sample that contains an appreciable number of stars with thick-disk kinematics, but lying in the ranges of metallicities and relative magnesium abundances extending to their solar values. Indeed, according to the isochrone ages, these stars are no older than the Sun. The angular momentum difference between the halo and the thick disk suggests the absence of a smooth transition between these two subsystems (Wyse and Gilmore 1992). A long delay of star formation after the mass supernova explosions, an active phase in the evolution of the Galaxy (see Berman and Suchkov 1991), could be responsible for this difference. This explanation simultaneously resolves the contradiction with the short time scale of free collapse of the protogalactic cloud $(\approx 0.4 \mathrm{Gyr})$, and the age at which the mass formation of thick-disk stars begins could prove to be even older than 1 Gyr. In other words the delay of star formation lengthens the time frames of the natural processes of collapse and chemical enrichment of the interstellar medium.

The vertical gradients in metallicity and magnesium abundance that we found as well as the correlations between the magnesium abundances (and, possibly also metallicities) and the perigalactic distances and the magnesium abundances and the orbital eccentricities naturally fit into this picture. However the question arises as to whether the stars with thick-disk kinematics, but lying in low orbits belong to the subsystem, since, if they do not belong to the thick disk there are no vertical gradients in it. In this case, the model of the subsystems formation through the interaction of the Early Galaxy with its satellites becomes of current interest. The stars of the so-called metal-poor thick-disk tail do not follow the patterns related to $[\mathrm{Fe} / \mathrm{H}]$ and exhibited by all the remaining stars of the subsystem. In addition, they show an anomalously large spread in $[\mathrm{Mg} / \mathrm{Fe}]$. Therefore, we believe that they were most likely formed inside isolated interstellar clouds that were enriched with chemical elements according to scenarios different from the evolution of the actively mixing main body of the interstellar matter of a single protogalactic cloud. Further studies and data on the abundances of other chemical elements in the subsystems stars are required to refine the thick-disk formation time scale and model.

In the next papers, we are going to consider the properties of stars in other Galactic subsystems and to further supplement the existing database of stars in the solar neighborhood with published determinations of the abundances of various chemical elements.

Acknowledgements: We are grateful to the anonymous referee for valuable remarks that forces us to present our results in a more reasoned way.

\section{References}

[1] C. Allen, A. Santillan, Rev.Mex. Astron. Astrophys, 22, 255, (1991)

[2] M. Barbier-Brossat, P. Figon, Astron. Astrophys Sup.Ser, 142, 217 (2000)

[3] T. Bensby, S. Feldsing and I. Lundstrem Astron. Astrophys. 410, 527 (2003) 
[4] B.G. Berman, A.A. Suchkov, Astrophys.Space Sci. 184, 169 (1991)

[5] J. Bernkopf, A. Fiedler and K. Fuhrmann, in Astrophysical Ages and Times Scales, Ed. by T. von Hippel, Ch. Simpson, and N. Manset (Astron.Soc.Pac.San Francisco, 2001), Astron.Soc.Pac.Conf.Ser. 245207 (2001).

[6] T.V. Borkova, V.A. Marsakov, Astron.Zh.77 750 77, 750 (2000) [Astron.Rep.44 665 (2000)].

[7] T. V. Borkova and V. A. Marsakov Astron.Zh.79, 510 (2002) [Astron.Rep.46 460 (2002)]

[8] T. V. Borkova and V. A. Marsakov Astron.Zh. 82, 453 (2005) [Astron.Rep.49 405 (2002)]

[9] A. Burkert, J.W. Truran and G. Hensler, Astrophys.J. 392, 651 (1992)

[10] C. Chiappini, F. Matteycci, R.G. Gratton, Astrophys.J. 477, 765 (1997)

[11] O.J. Eggen, D. Linden-Bell, A. Sandadge, Astrophys.J. 136, 765 (1962)

[12] O.J. Eggen Astron. J. 115, 2397 (1998)

[13] S. Feldzing, T. Bensby, I. Lundstrom, Astron. Astrophys. 397, L1 (2003)

[14] K. Fuhrmann, M. Axer, T. Gehren, Astron. Astrophys. 301, 222 (1995)

[15] K. Furmann, Astron. Astrophys. 338, 183 (1998)

[16] K. Fuhrmann, The First Stars. Proc. MPA/ESO Workshop, A. Weiss, T.G. Abel, V. Hill (eds), Springer, p. 68 (2000)

[17] R. Gratton, E. Carretta, F. Matteucci, C. Sneden, Astron. Astrophys, 358, 671 (2000)

[18] G. Gilmore, R.F.G. Wyse, in The Galaxy, ed. G. Gilmore \& B. Carswell (NATO ASI Ser. . 207) (Dordrecht: Reidel), 247 (1987)

[19] P. Kroupa, Mon.Not.Rou.Astron.Soc. 330, 707 (2002)

[20] F. Matteucci, I. Greggio, Astron. Astrophys. 154, 279 (1986)

[21] F. Matteucci, The Chemical Evolution of the Galaxy, Astrophys. and Spase Sci. Library 253, Kluwer Acad. Publ. (2001)

[22] V. A. Marsakov and A. A. Suchkov Astron.Zh. 54, 1232 (1977) [Sov.Astron.21 700 (1977)].

[23] V. A. Marsakov and Yu. G. Shevelev Astron.Zh. 72, 321 (1995) [Astron.Rep. 39287 (1995)].

[24] L. Mashonkina, T. Gehren, C. Travaglio, T. Borkova, Astron. Astrophys. 397, 275 (2003)

[25] J.F. Navarro, A. Helmi, K.C. Freeman Astrophys. J. 601, L43 (2004)

[26] D.L. Nidever, G.W. Marcy, R.P. Butler, et al., Astrophys. J.Sup.Ser. 141, 503 (2002)

[27] B. Nordstrem, M. Mayor, J. Andersen et al., Astron. Astrophys. 418, 989 (2004) 
[28] J. Norris, M. Bessel, A.J. Piscles, Astropys.J. Supl.Ser. 58, 463 (1985)

[29] J.X. Prochaska, S.O. Naumov, B.W. Carney, A. McWilliam, A.M. Wolte Astron.J. 120, $2513(2000)$

[30] P.J. Quinn, L. Hernquist, D.P. Fullagar, Astropys.J. 403, 74 (1993)

[31] K.U. Ratnatunga, J.N. Bahcall, S. Casrtano, Astropys.J. 291, 260 (1989)

[32] A.C. Robin, M. Hauwood, M. Creze et al. Astron. Astrophys. 305, 125 (1996)

[33] N. N. Shimanskaya, L. I. Mashonkina, and N. A. Sakhibullin, Astron.Zh. 77, 599 (2000) [Astron.Rep. 44530 (2000)].

[34] C. Soubiran, O. Bienaume, A. Siebert, Astron. Astrophys. 398, 141 (2003)

[35] F.-K. Thielemann, M. Hashimoto, K. Nomotto, Astrophys.J. 349, 222 (1990)

[36] T. Tsujimoto, K. Nomoto, Y. Yoshii et al., Mon.Not.Roy.Astron.Soc. 277, 945 (1995)

[37] R.F.G. Wyse and G. Gilmore, Astron.J. 104, 144 (1992)

[38] R. Zinn, Astrophys. J. 293, 424 (1985) 

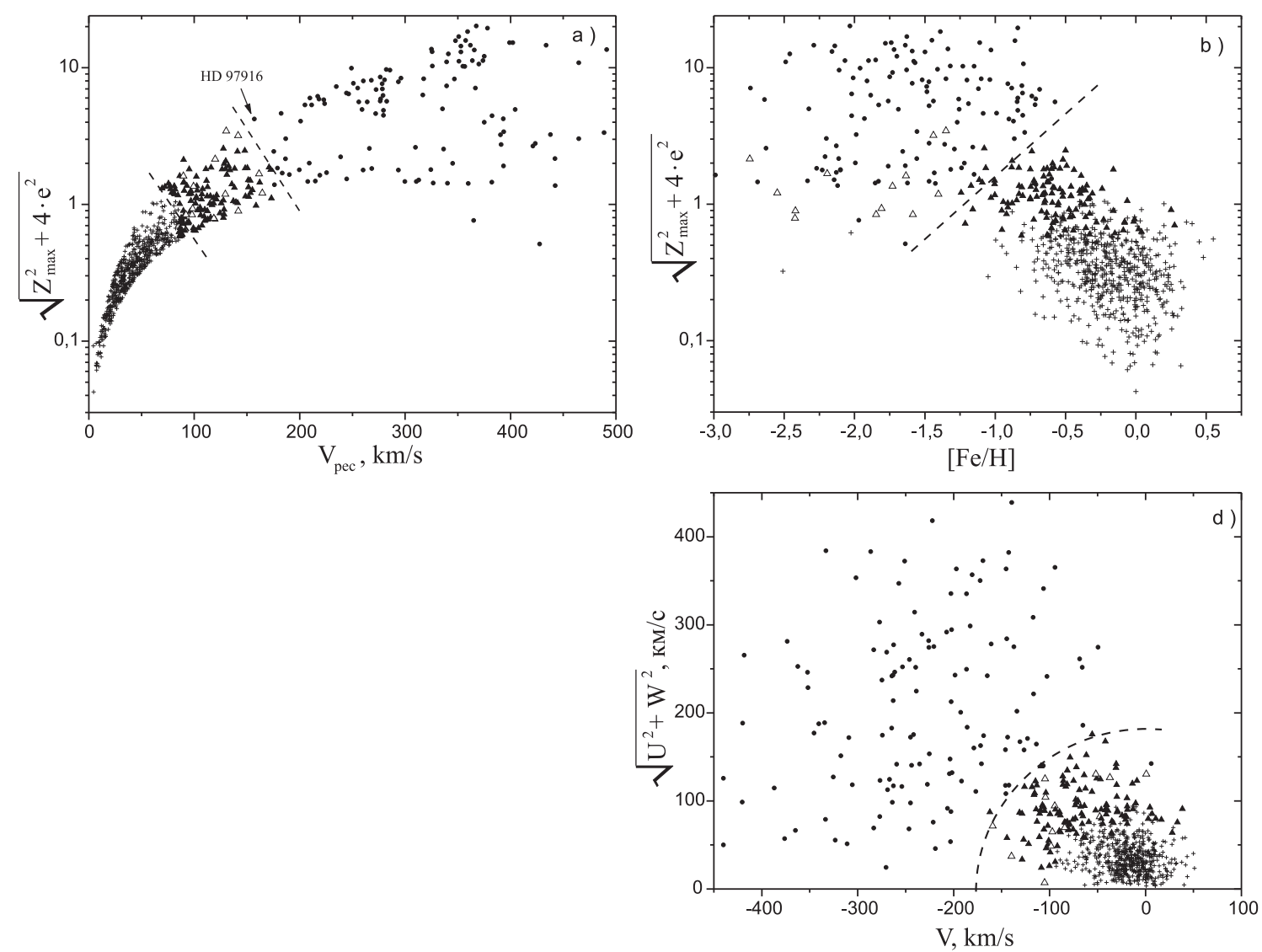

Figure 1: Relations between $\sqrt{Z_{\max }^{2}+4 \cdot e^{2}}$ and the residual stellar velocities with respect to the local standard of rest (a) metallicities (b), circular velocities (c) and between the residual stellar velocity components (d): crosses - thin-disk stars; filled triagles - thick-disk stars; open triagles - stars of the metal-poor thick-disk tail; filled circles - halostars; and dotted lines - demarcation lines drawn by eye. 


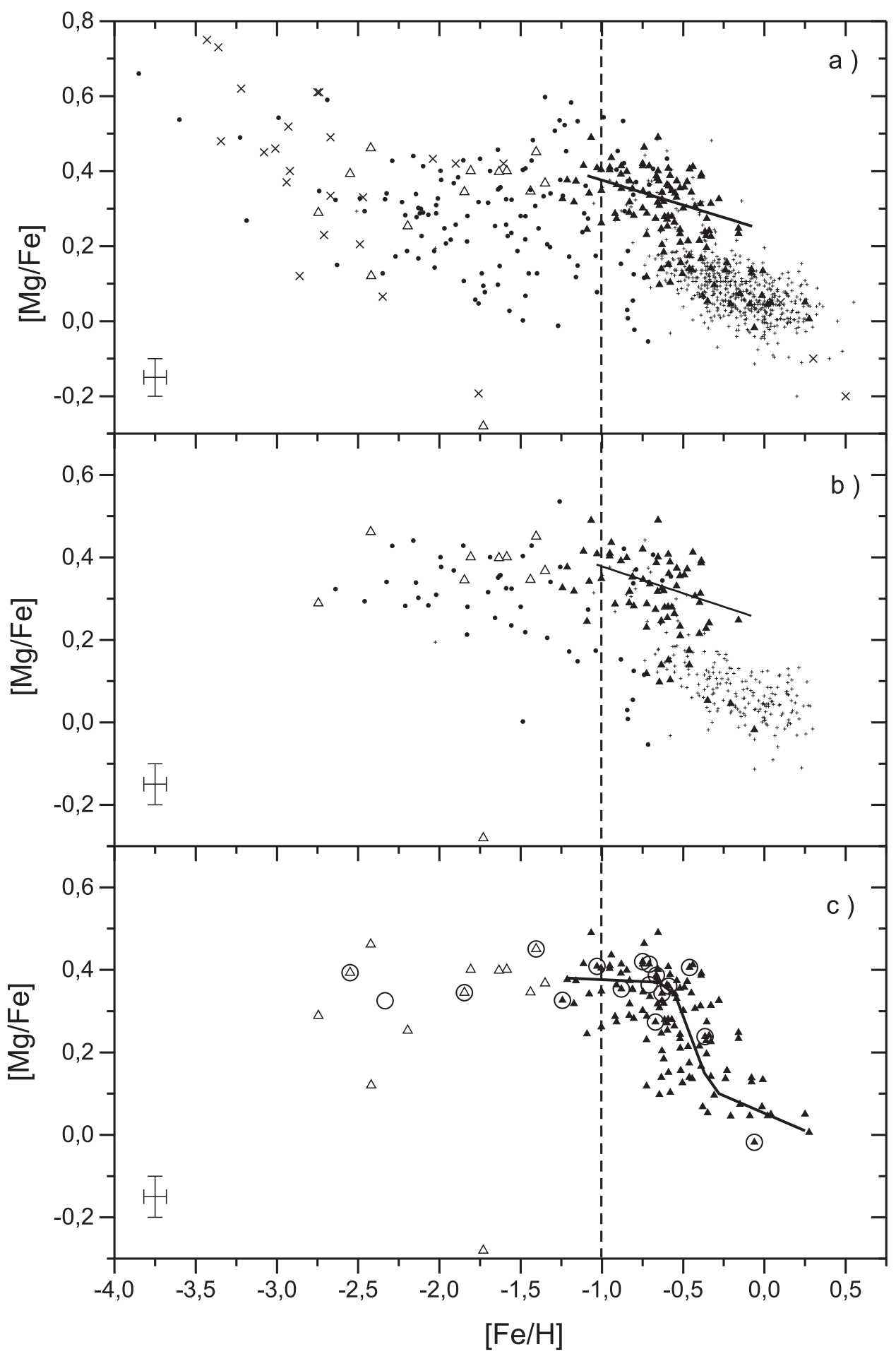

Figure 2: Relations between metallicity and relative magnesium abundance (a) for all stars of our catalog, (b) for the stars in which the magnesium abundances were averaged over several sources, and (c) only for the thick-disk stars. The notation is the same as that in Fig. 1; the crosses denote the stars with unmeasured radial velocities. The vertical dotted lines indicate the point at which the knee of the $([\mathrm{Mg} / \mathrm{Fe}]-[\mathrm{Fe} / \mathrm{H}])$ relation begins, and the inclined straight lines represent the regression lines for metal-rich and, simultaneously magnesium-rich thick-disk stars; the broken curve represents the median line drawn by eye. The open circles in the panel denote the stars of the Arcturus moving group. The error bars are shown. 

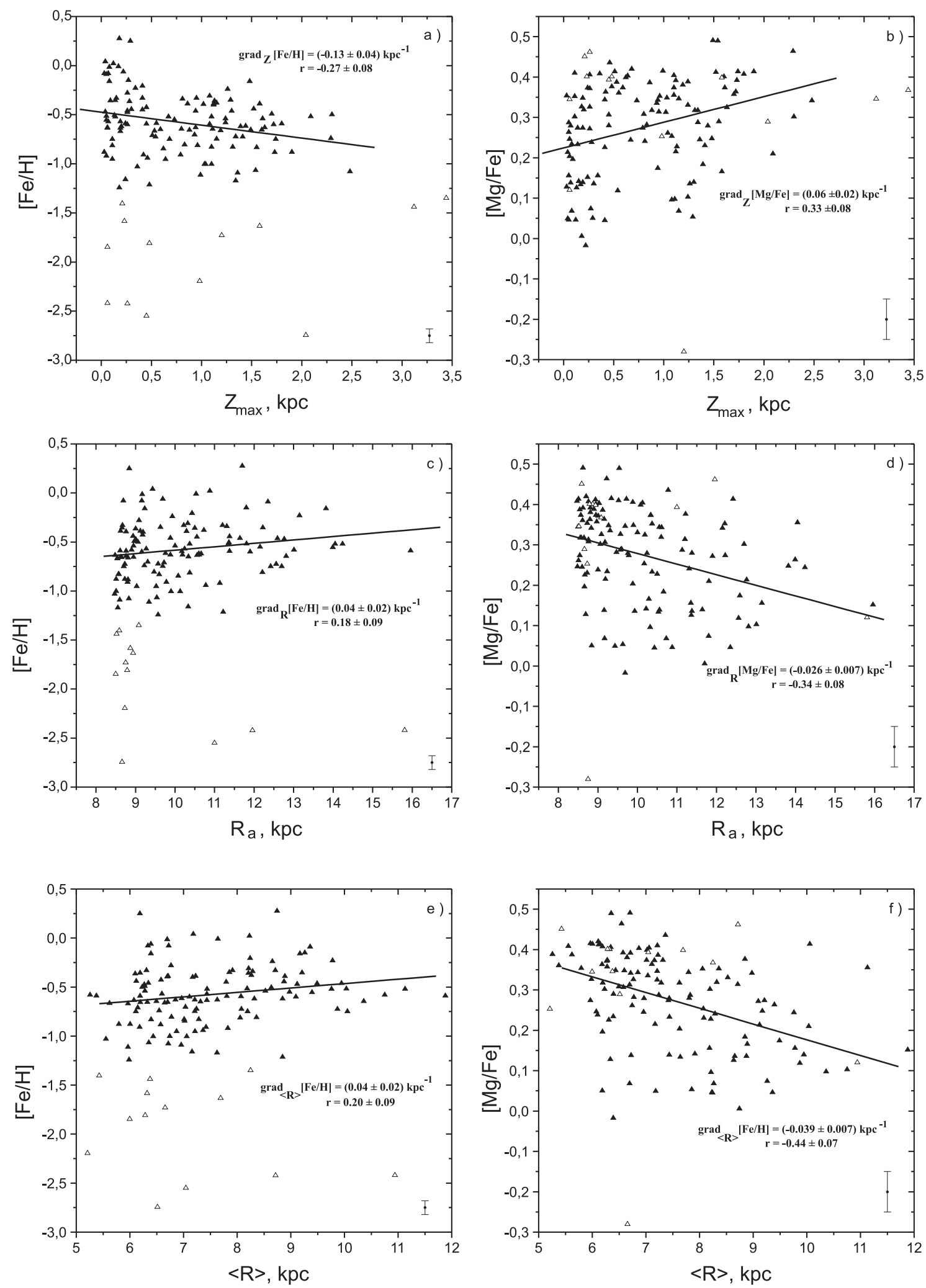

Figure 3: Relations of the metallicity and relative magnesium abundance in the atmospheres of thick-disk stars to their maximum distance fromthe Galactic plane (a), (b), to the apogalactic distance (c), (d), and to the mean orbital radius (e), (f). The notation is the same as that in Fig. 1. The solid lines represent the regression lines for the thick-disk stars. The gradients and correlation coefficients are shown. The error bars are given. 

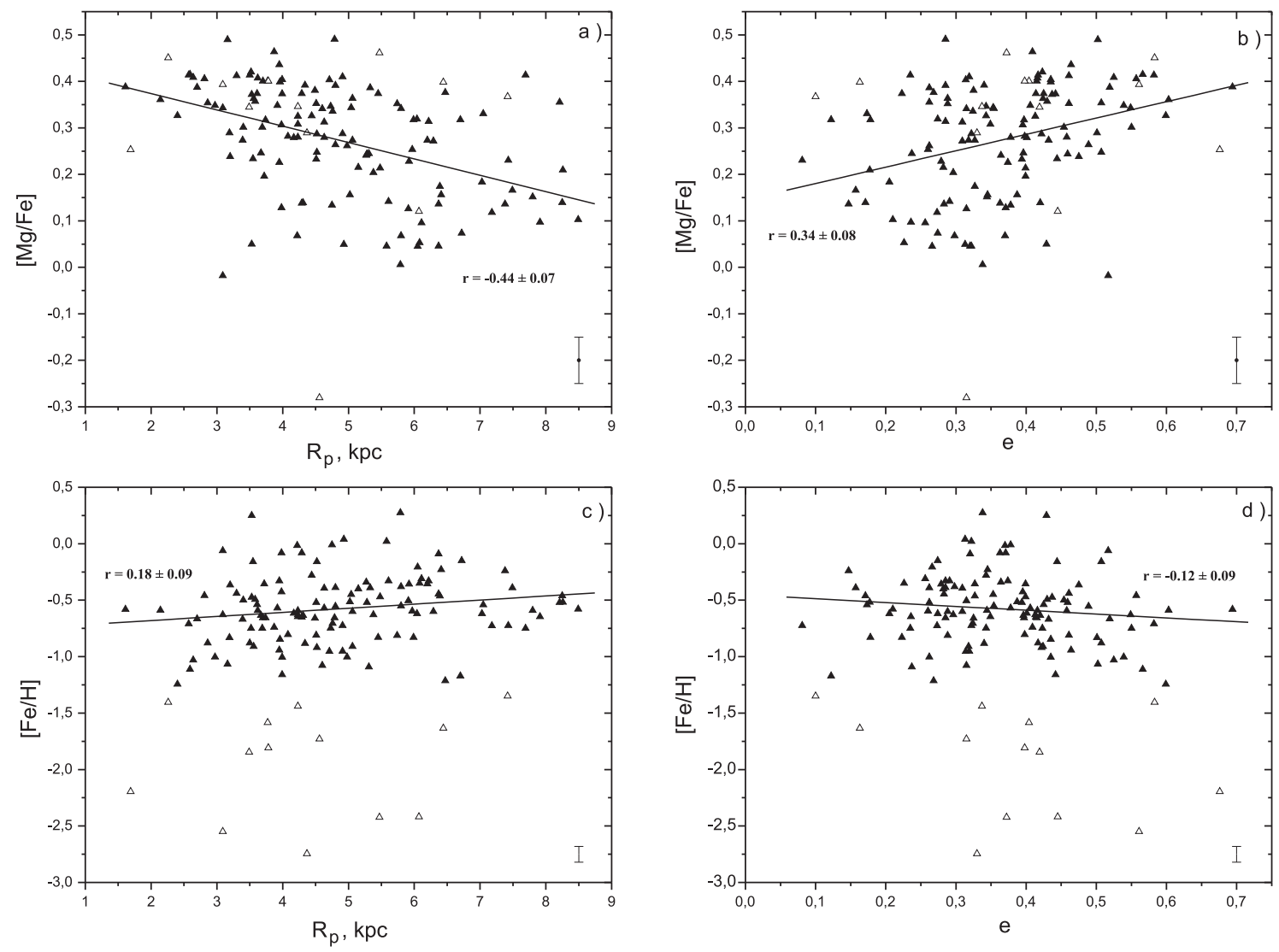

Figure 4: Relations of the metallicity and relative magnesium abundances in the thickdisk stars to their perigalactic distances (a), (c) and orbital eccentricities (b), (d). The correlation coefficients are shown. The error bars are given. 

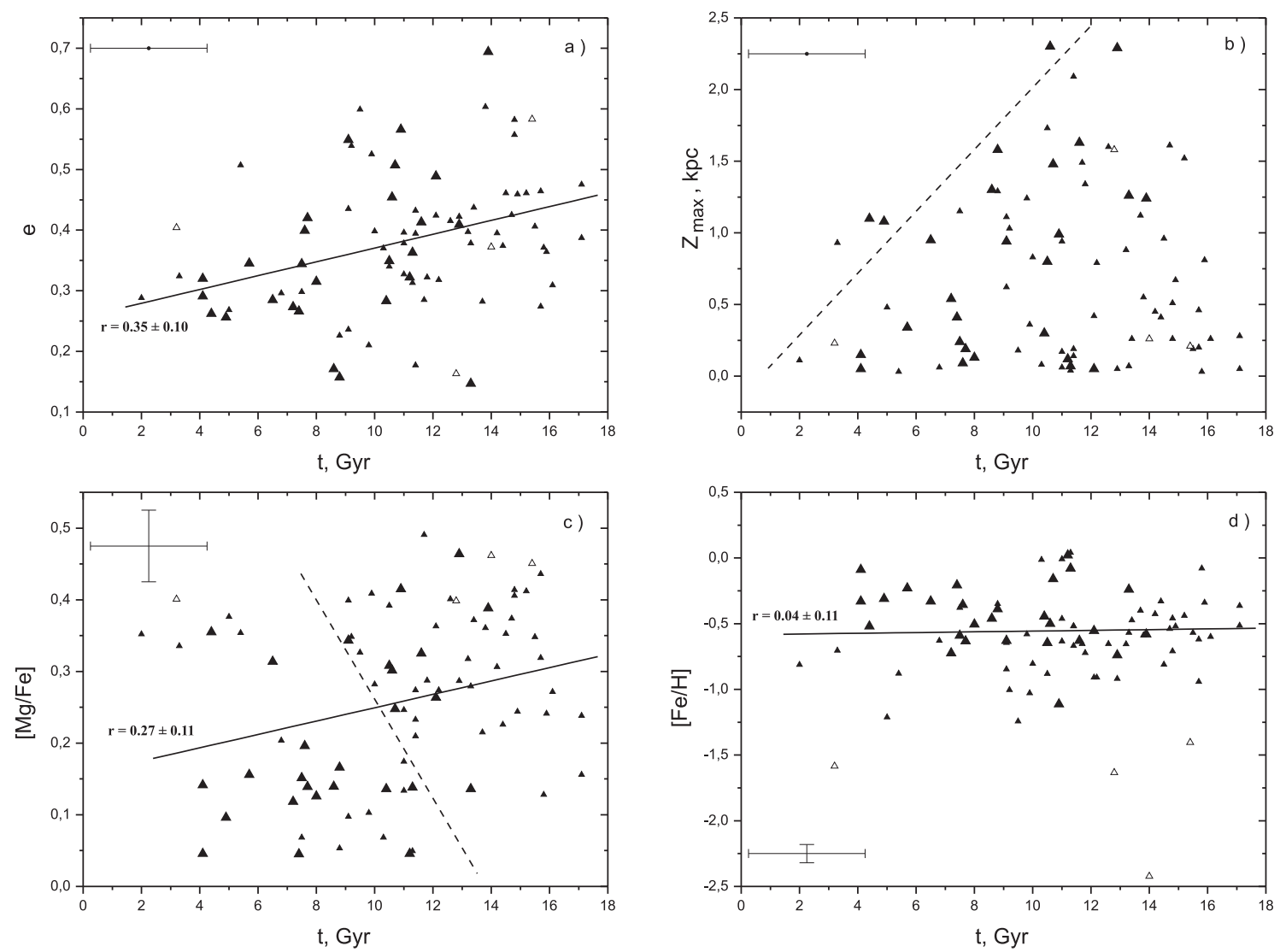

Figure 5: Relations of the orbital eccentricities (a), the maximum distances of orbital points from the Galactic plane (b), and the relative magnesium abundances (c) and metallicities (d) in thick-disk stars to the age. The larger triangles denote the stars with age errors of less than 2 Gyr. The solid lines represent the regression lines. The correlation coefficients are shown. See the text for an explanation of the dotted lines in panels (b) and (c). The error bars are given. 

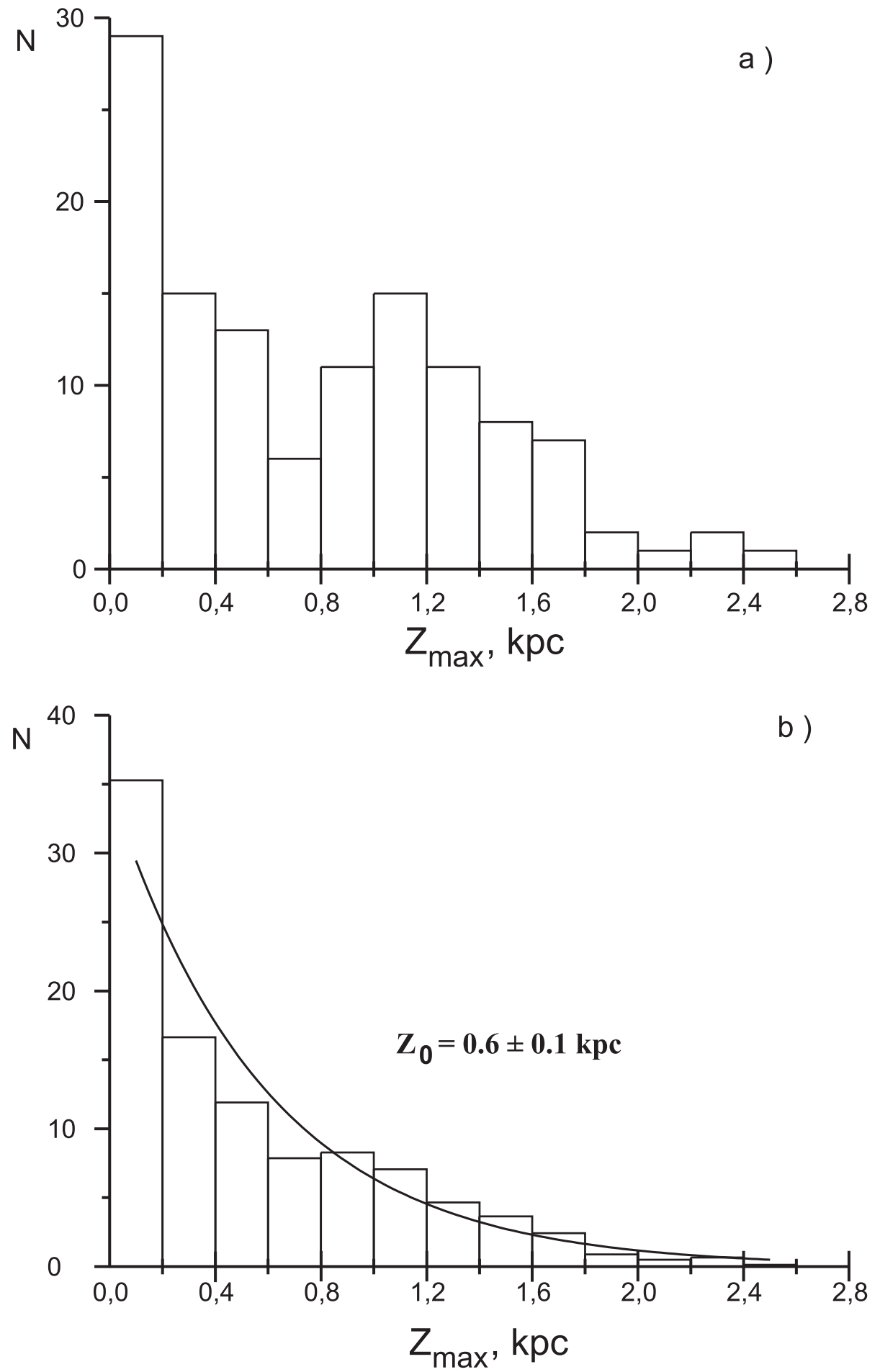

Figure 6: Distribution in maximum distance from the Galactic plane and (b) reconstructed $\mathrm{Z}$ distribution for thick-disk stars. The solid curve indicates the exponential fit to the distribution, and the scale height is indicated together with its uncertainty. 